\title{
Selective-Mapping Type Peak Power Reduction Techniques For Turbo Coded OFDM
}

\author{
Mao-Chao Lin \\ Department of Electrical Engineering \\ National Taiwan University \\ Taipei 106, Taiwan, R.O.C. \\ Telephone: (886) 223635251 ext. 323 \\ Fax: (886) 223638247 \\ Email: mclin@cc.ee.ntu.edu.tw
}

\author{
Yung-Chih Tsai and Chung-Jay Yang \\ Graduate Institute of Communication Engineering \\ National Taiwan University \\ Taipei 106, Taiwan, R.O.C. \\ Email: d92942009@ntu.edu.tw \\ r91942083@ntu.edu.tw
}

\begin{abstract}
In an earlier paper, a selective-mapping type turbo coded OFDM scheme was proposed, for which the interleaver of the encoder of the turbo coding can be varied to reduce the associated PAPR (peak to average power ratio) while the transmission of side information is not needed. In this paper, we propose two modified versions of that scheme. For the first, the variation of the interleaver of the turbo encoder is replaced by the variation of the bit permutation of the turbo codeword. For the second, the variation of the interleaver of the turbo encoder is replaced by the variation of a maximum-length sequence which is added to the turbo codeword. These two modified schemes can provide better PAPR reduction capability.
\end{abstract}

\section{INTRODUCTION}

Orthogonal frequency division multiplexing (OFDM) is a popular modulation choice for nowadays wireless applications. The occurrence of high PAPR is a well-known disadvantage of the OFDM systems. A number of techniques have been proposed for the reduction of PAPR in the OFDM system, which are majorly divided into two classes, the distortion based techniques and the redundancy based techniques. The distortion based techniques such as clipping, and peak windowing [1], [2] directly suppress the time-domain signals for which the signal powers exceed a certain threshold. These techniques usually result in poor error performance and significant out-of-band power. The redundancy based technique including coding, selective-mapping, tone reservation, and tone injection [3], [4], [5], [6], [7], etc. The undesired effects occurring to the distortion techniques can be alleviated with the penalty of the reduced transmission rates due to the introduction of redundancy. The basic idea of selective-mapping (SLM) technique is to generate several OFDM symbols as candidates and then select the one with the lowest PAPR for actual transmission. Conventionally, the transmission of side information is needed so that the receiver can use the side information to tell which candidate is selected in the transmission and then recover the information.

In 2003, a selective-mapping scheme for turbo coded OFDM which does not need information [8] was proposed, which employs the discriminating characteristics of the interleaver of the turbo coded system. Several distinct interleavers are used as candidates for the selection operations in the transmitter. The receiver uses the MAP decoder for the turbo code to calculate the reliability of each candidate. Although side information is not available, the reliability of the decoded results will be high and the receiver can recover the correct codeword in case that the interleaver chosen by the receiver is correct. In case that the interleaver is not the right one, the reliability of the decoded results will be very low and the receiver needs to try another interleaver. The price to pay is the increased decoding complexity. Moreover, there is room for improving the capability of PAPR reduction. The reason is that we note that varying interleavers of turbo encoder will only vary the parity bits of the second component convolutional code of the turbo codes. With this observation, in this paper, we present two modified side-information-free selective mapping turbo coded OFDM schemes for which all the code bits of a whole turbo codeword may be varied so that the PAPR can be substantially reduced. Simulation results show that better PAPR reduction (measured by out-of band power) can be achieved for the modified schemes.

\section{A Selective-MAPping Turbo Coded OFDM SCHEME WITH SIDE INFORMATION}

Consider a selective mapping turbo coded OFDM scheme for which the transmitted side information is well protected. In this paper, it is denoted as scheme A. The idea is close to that of [9]. For the transmitter, the message sequence $\bar{u}$ is scrambled by $Q$ distinct scramblers. The $i$-th scrambler adds the input $\bar{u}$ to a sequence $\bar{t}_{i}$ generated by the $i$-th $m$-sequence generator to yield the scrambled output $\bar{u} \oplus \bar{t}_{i}$. The sequence obtained by padding $\left\lceil\log _{2} Q\right\rceil$-bit side information $\bar{s}_{i}$ to $\bar{u} \oplus \bar{t}_{i}$ is then processed through turbo encoding, signal mapping, and IFFT to yield an output sequence $\bar{a}_{i}$ for each $i$. Finally, the sequence with lowest peak power among the $Q$ output sequences, i.e., $\bar{a}_{1}, \bar{a}_{2}, \cdots, \bar{a}_{Q}$ is selected for transmission. In the SLM receiver, the turbo decoder yields $\bar{s}^{\prime}$ together with $\bar{u}^{\prime} \oplus \bar{t}^{\prime}$. With the side information $\bar{s}^{\prime}$, we can identify the associated $m$-sequence $\bar{t}^{\prime}$. By adding $\bar{t}^{\prime}$ to $\bar{u}^{\prime} \oplus \bar{t}^{\prime}$, we can recover the message $\bar{u}^{\prime}$. The transmitter and receiver structures are shown in Fig. 1 and Fig. 2 respectively, where $Q=16$. 


\section{A KNOWn SElective-MapPing Turbo CODED OFDM SCHEME WITHOUT SIDE INFORMATION}

A selective-mapping type without side information turbo coded OFDM system, denoted scheme B, has been presented in [8]. The transmitter is shown in Fig. 3, where $Q$ distinct interleavers are used. For $i=1,2, \cdots, Q$, the message sequence $\bar{u}$ was encoded by the turbo code using the $i$-th interleaver to yield a turbo coded sequence $\bar{v}_{i}$, which is mapped to a signal point $A_{i}$ and then is converted into $\bar{a}_{i}$ through the IDFT operation. Then, $\bar{a}_{j}$ is selected for transmission if $\bar{a}_{j}$ has PAPR no greater than any $\bar{a}_{i}, i \neq j$. The structure of the receiver is shown in Fig. 4, where $\bar{r}$ represents the received vector. There are $Q$ decoders corresponding to $Q$ interleavers respectively. At the $r$-th iteration, $1 \leq r \leq I$, the $i$-th decoder computes the reliability $\tilde{L}_{i}^{r}\left(d_{k}\right)=\log \left[\operatorname{prob}\left(d_{k}=1 \mid \bar{r}\right) / \operatorname{prob}\left(d_{k}=0 \mid \bar{r}\right)\right]$, for the $k$-th message bit $d_{k}$. At the $S$-th iteration of decoding, $\beta_{i}=\sum_{k} \tilde{L}_{i}^{S}\left(d_{k}\right)$ is computed for each $i$, where $1 \leq S \leq I$, . The $j$-th decoder is selected if $\beta_{j}$ is no less than any $\beta_{i}$ for $i \neq j$. Only the $j$-th decoder needs to continue its decoding until the $I$-th iteration and takes the hard decision on $\tilde{L}_{j}^{I}$ as the decoded output. Note that in scheme B, transmission of side information is not needed. The complexity of the receiver is $[(Q-1) S+I] / I$ times of that of a conventional turbo decoder.

\section{New SElEctive-MAPPING TURbo Coded OFDM SCHEME WITHOUT SIDE INFORMATION}

From Fig. 3, we can easily note that the distinct interleavers for the turbo encoders will result in turbo codewords for which only the parity parts of RSC 2 (the second component convolutional code) will be different while the message parts and the parity parts of RSC 1 (the first component convolutional code) are identical . This may restrict the capability of PAPR reduction. In this section, we propose two modified schemes for which the whole turbo codeword may be varied for each selection.

Consider the first modified scheme, denoted scheme $\mathrm{C}$, for which the transmitter is shown in Fig. 5, where $Q$ distinct interleavers are used to permute the code bits of the turbo codeword $\bar{v}$. Using the $i$-th interleaver, the bit positions of the turbo codeword $\bar{v}$ were permuted to result in a sequence $\bar{v}_{i}$, which is then converted into $\bar{a}_{i}$ through the IDFT operation. Now we have $Q$ candidates, i.e., $\bar{a}_{1}, \bar{a}_{2}, \cdots, \bar{a}_{Q}$. The transmitter selects the candidate with the lowest PAPR for transmission. In the receiving end, $Q$ distinct deinterleavers are applied to the received frame to yield $Q$ candidates, $\bar{r}_{1}, \bar{r}_{2}, \cdots$, $\bar{r}_{Q}$. The turbo decoder will decode each $\bar{r}_{i}, i=1,2, \cdots, Q$. The reliability of decoding each $\bar{r}_{i}$ is calculated in a manner similar to that in scheme $\mathrm{B}$. If $\bar{r}_{j}$ yields the largest reliability at the $S$-th iteration, the decoder will continue its decoding process until the $I$-th iteration and then use the decoded results as the final output.

Consider the second modified scheme, denoted scheme $\mathrm{D}$, for which the transmitter is shown in Fig. 6, where $Q$ maximum-length sequences (m-sequences) are used to scramble the turbo codeword $\bar{v}$. The $i$-th maximum length sequence $\bar{t}_{i}$ is added to the turbo codeword $\bar{v}$ to result in a sequence $\bar{v}_{i}$ $=\bar{v} \oplus \bar{t}_{i}$, which is then converted into $\bar{a}_{i}$ through the signal mapper and the IDFT operation. We have $Q$ candidates, i.e., $\bar{a}_{1}, \bar{a}_{2}, \cdots, \bar{a}_{Q}$. The transmitter selects the candidate with the lowest PAPR for transmission. The decoders of either scheme $\mathrm{C}$ or scheme $\mathrm{D}$ can be designed in a manner (and complexity) similar to that of scheme B. Hence, no side information is needed for either scheme $\mathrm{C}$ or scheme $\mathrm{D}$.

\section{Simulation Results}

Consider the case of 256-subcarrier BPSK OFDM, which uses a rate $1 / 2$ turbo code of length 256 . The turbo encoder is formed by two 4-state recursive systematic convolutional (RSC) codes. These RSC elementary encoders have the same polynomial generators $(7,5)$. In this simulation, iteration number $I=7$ is used. Let candidate number $Q=$ 16. We perform simulation for schemes A, B, C, D and the turbo-coded OFDM without using any PAPR reduction technique respectively, where oversampling factor of 4 is used. In the transmitter end, a power amplifier with $6 \mathrm{~dB}$ clipping (backoff) is used. For scheme B, the 16 interleavers [10] are generated by the mapping function $\Omega: c_{m} \rightarrow c_{m+1(\bmod N)}, 0 \leq m<N$,

where

$c_{m} \equiv \frac{k m(m+1)}{2}(\bmod N), 0 \leq m<N$

$N$ is the interleaver size and $k$ is a odd integer. In the simulation for scheme $\mathrm{B}$, we choose $k=$ $\{35,37,39,41,45,49,57,59,69,71,81,87,89,103,107,121\}$ when interleaver size $N=128$. For scheme C, the 16 interleavers are generated by the same $k$ value, but now the size of interleaver is 256 . For scheme A and D, we randomly choose sixteen sequences from $255(255,8,128)$ maximum length sequences with period of 255 as $\bar{t}_{1}, \bar{t}_{2}, \cdots, \bar{t}_{16}$.

We use $10^{5}$ OFDM symbols and CCDF (Complementary Cumulative Density Function) to evaluate the PAPR reduction capability in Fig. 7. From Fig. 7, we see that scheme A and scheme D have the same PAPR reduction capabilities. Peakto-average power (PAP) greater than $7 \mathrm{~dB}$ are not observed for schemes $A$ and $D$ and hence the associated CCDF curve for PAP beyond $7 \mathrm{~dB}$ is represented by a vertical dashed line. In case of specific soft-limiter for the power amplifier, we may also evaluate the PAPR reduction capability by PSD (power spectral density). In Fig. 8, the PSD of rate $1 / 2$ turbo coded OFDM systems are given, where $6 \mathrm{~dB}$ soft-limiter is considered. We see that scheme A and scheme D have the lowest out-of-band power, while scheme $C$ is close. Schemes A, C and D have PAPR reduction capability better than scheme B. This phenomenon conforms to our expectation that each of scheme A, C and D varies the whole turbo codeword in the operation of selective mapping while scheme $\mathrm{B}$ varies only a small portion of the turbo codeword in the operation of selective mapping. The error performances of turbo coded OFDM systems over AWGN channel are given in the biterror-rate (BER) curves in Fig. 9, where the ideal turbo coded OFDM means the system does not consider the usage of both 
PAPR reduction and soft limiter, while turbo coded OFDM with clipping $6 \mathrm{~dB}$ means the systems does not consider the usage of PAPR reduction but consider the usage of a soft-limiter with $6 \mathrm{~dB}$ clipping. Before error performance is measured, we first use $S=6$ iterations to judge which candidate is the most possible one. Then in the 7th iteration ,the error rate is made. We see that schemes B, C, and D have similar error performances at high signal-to-noise (SNR) conditions and are better than scheme A. The inferior performance of scheme A is due to the fact that a wrong side information at the receiver side will degrade the error performance. In particular, for high SNR, schemes B, C, and D have performance similar to that of turbo coded OFDM without using any PAPR reduction technique.

\section{CONCLUSION}

We propose two new side-information free selectivemapping type turbo coded OFDM schemes. Compared to the previous scheme in [8], the new schemes have similar complexity, similar BER performance at high SNR and better PAPR reduction capability. Compared to the scheme using side information, the side-information free schemes have better BER performances and have higher transmission rate.

\section{REFERENCES}

[1] R. van Nee and A. de Wild, "Reducing the peak-to-average power ratio of ofdm," IEEE Vehicular Technology Conferences, vol. 3, pp. 20722076, May 1998.

[2] X. Li and L. C. Jr., "Effects of clipping and filtering on the performance of ofdm," IEEE Commun. Lett., vol. 2, no. 5, pp. 131-133, May 1998.

[3] R. Bauml, R. Fisher, and J. Huber, "Reducing the peak-to-average power ratio of multicarrier modulation by selected mapping," Electronics Letters, vol. 32, no. 22, pp. 2056-2057, Oct. 1996.

[4] P. V. Etvelt, G. Wade, and M. Tomlinson, "Peak to average power reduction for ofdm schemes by selective scrambling," Electronics Letters, vol. 32, no. 21, pp. 1963-1964, Oct. 1996.

[5] A. D. S. Jayalath and C.Tellambura, "The use of interleaving to reduce the peak-to-average power ratio of an ofdm signal," Proc. IEEE GLOBECOM 2000, vol. 1, pp. 82-86, Nov. 2000.

[6] D. J. G. Mestdagh and P. M. P. Spruyt, "A method to reduce the probability of clipping in dmt-based transceivers," IEEE Trans. Commun., vol. 44, no. 10, pp. 1234-1238, Oct. 1996.

[7] J. Tellado, Multicarrier modulation with low PAR - Applications to DSL and Wireless. Kluwer Academic Publishers, Jan. 2000.

[8] M. C. Lin, K. C. Chen, and S. L. Li, "Turbo coded ofdm system with peak power reduction," IEEE Vehicular Technology Conferences, vol. 4, pp. 2282-2286, Oct. 2003.

[9] M. Breiling, S. H. M. Weinfurtner, and J. B. Huber, "SLM peak-power reduction without explicit side information," IEEE Commun. Lett., vol. 5, pp. 239-241, June 2001.

[10] O. Y. Takeshita and J. D. J. Costello, "New deterministic interleaver designs for turbo codes," IEEE Trans. Inform. Theory, vol. 46, no. 6, pp. 1988-2006, Sept. 2000.

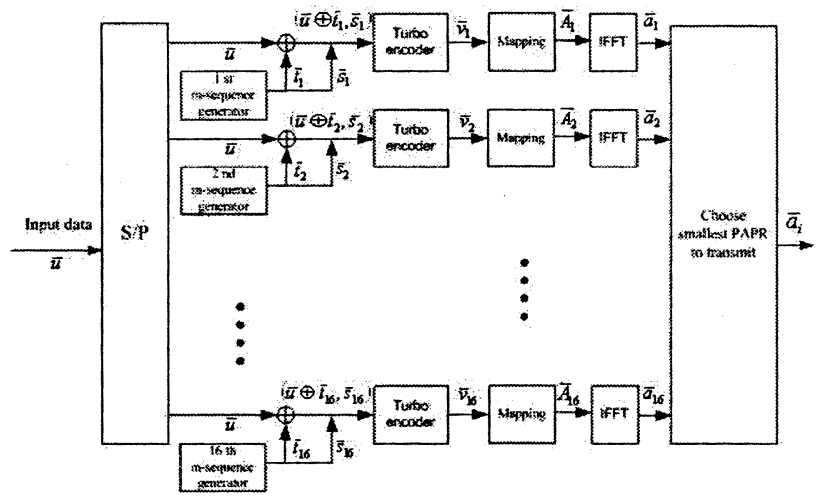

Fig. 1. The transmitter of Scheme A.

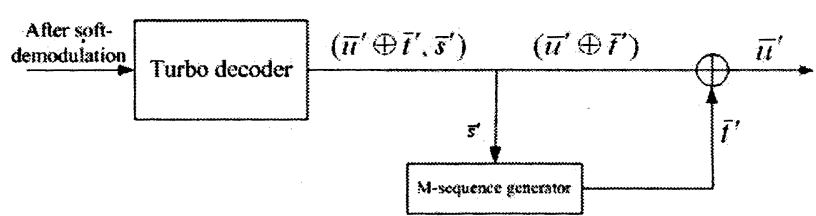

Fig. 2. The receiver of Scheme A.

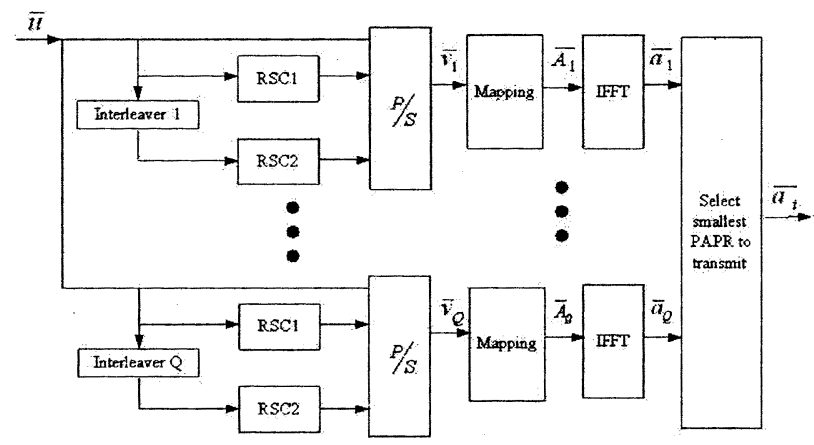

Fig. 3. The transmitter of Scheme B.

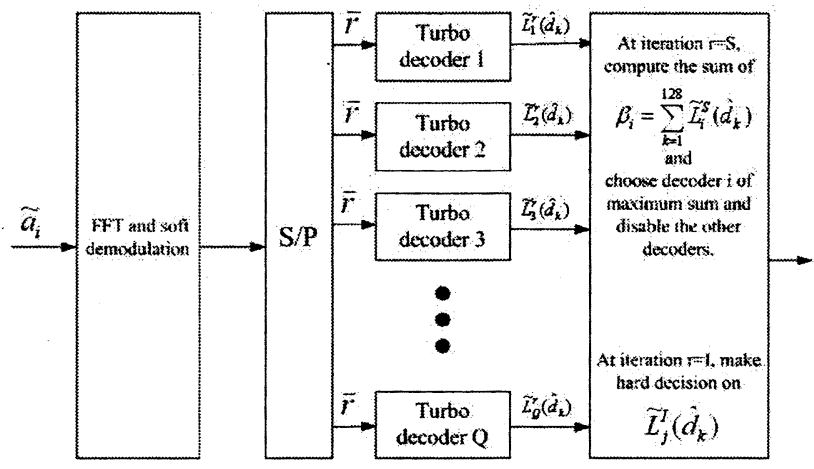

Fig. 4. The receiver of Scheme B. 


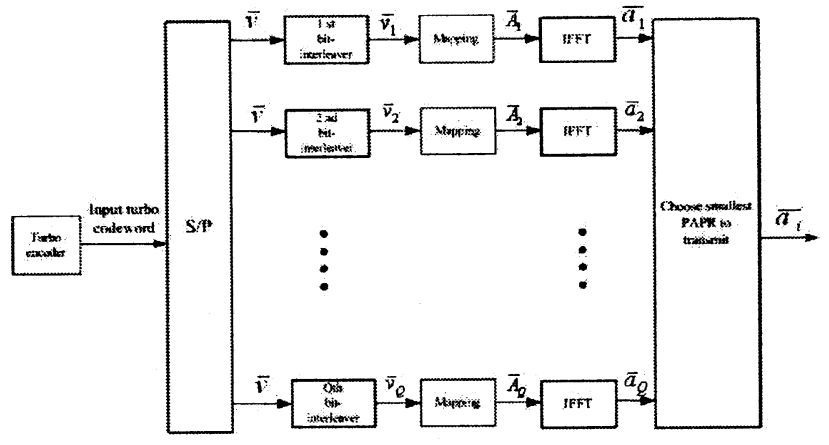

Fig. 5. The transmitter of Scheme C.

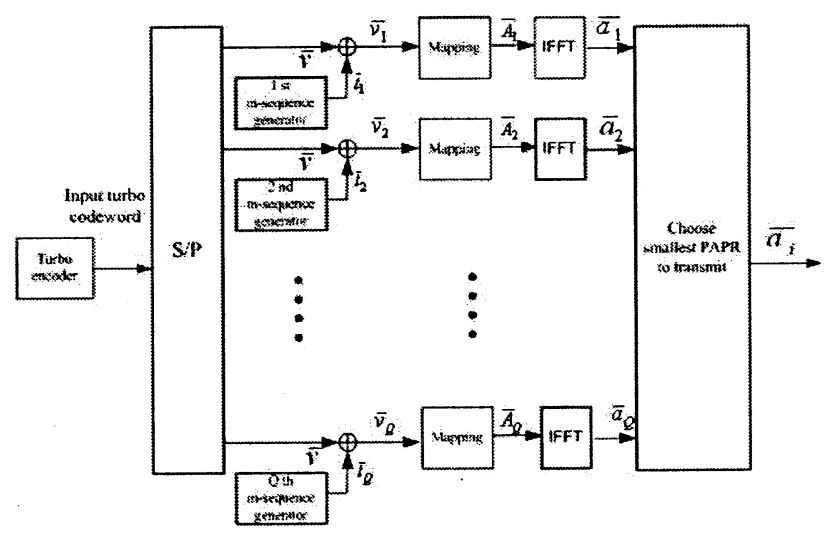

Fig. 6. The transmitter of Scheme D.

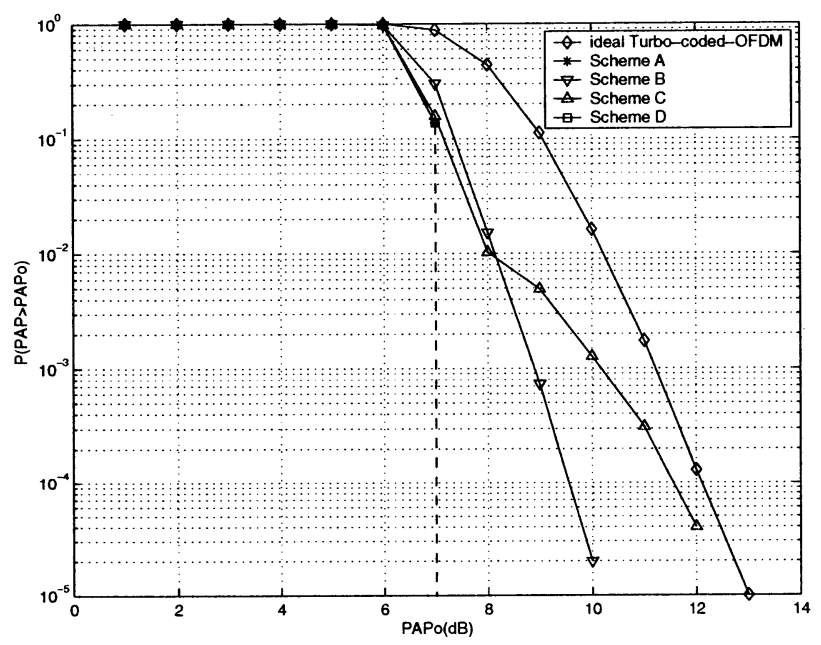

Fig. 7. CCDF of rate $1 / 2$ BPSK Turbo-coded systems

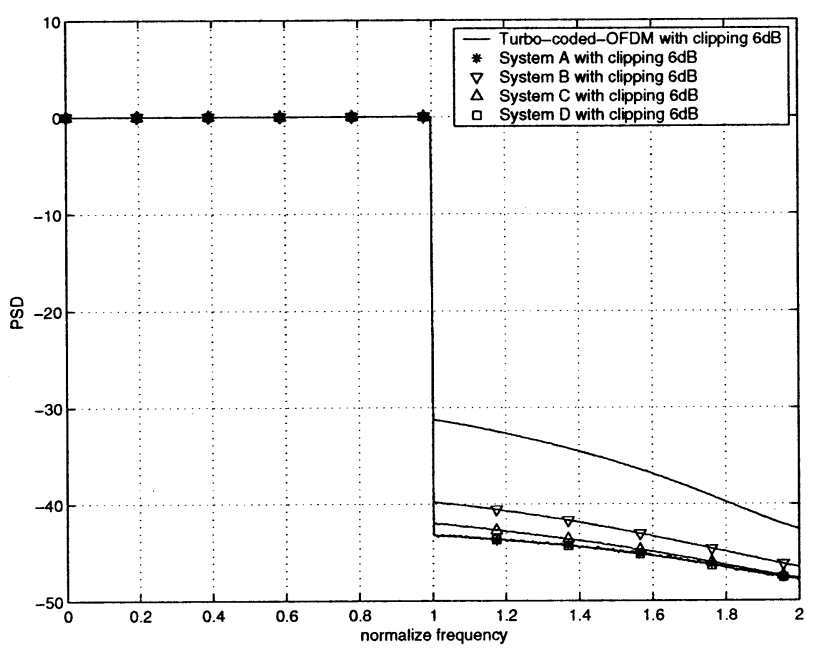

Fig. 8. PSD for rate 1/2 BPSK Turbo-coded OFDM systems

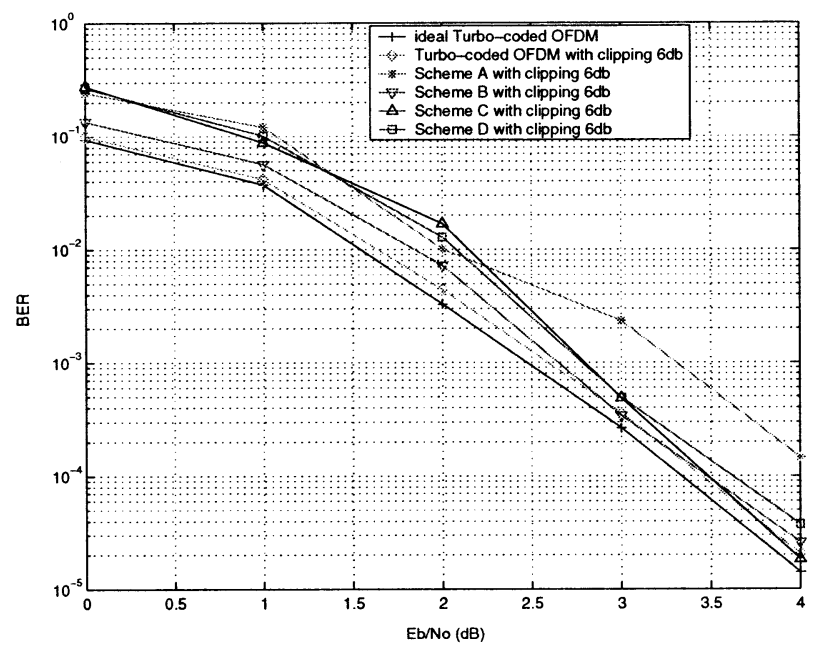

Fig. 9. BER for rate 1/2 BPSK Turbo-coded OFDM systems 